\title{
The determination of urinary 17-ketosteroids by an improved Zimmermann reaction
}

\author{
R. N. BEALE, J. O. BOSTROM, AND D. CROFT \\ From the Institute of Clinical Pathology and Medical Research, \\ Department of Public Health, Sydney, Australia ${ }^{1}$
}

SYNOPSIS Improvements in the Zimmermann reaction are effected by using methanol as a solvent for the reagents employed in the reaction in place of ethanol or aqueous ethanol. The advantages are as follows. Considerably lower blanks are obtained; extraneous chromogens are negligible at the wavelength of measurement, so that no correction procedure or extraction process is required; high concentrations of potassium hydroxide may be used at an elevated temperature leading to good sensitivity and a considerably shortened reaction time; and finally, the potassium hydroxide is stable for long periods under good storage conditions. A steroid with keto (oxo) groups at positions 3,11 , and 20 , but not at 17 , failed to give any coloured product.

The reaction between nitro-aromatic compounds and ketones or aldehydes in the presence of alkali appears to have been first recorded by Janovsky in 1886 and amplified by von Bittó in 1892 (see Hickinbottom, 1936, for references). Later, the reaction was applied to ketosteroids by Zimmermann $(1935,1936)$ and subsequently a colorimetric method for assaying urinary 17-ketosteroids was evolved by Callow, Callow, and Emmens (1938). Since then, what is now referred to as the Zimmermann, or sometimes the Callow-Zimmermann, reaction has received much attention as a means of determining urinary 17-keto-, 17-ketogenic, and 17-hydroxycorticosteroids when used in conjunction with the elegant methods primarily due to Norymberski (1952) and developed by Norymberski, Stubbs, and West (1953) and by Appleby, Gibson, Norymberski, and Stubbs (1955).

The difficulties involved in the practical application of the Zimmermann reaction are well appreciated, and a discussion of the main problems arising has been published recently by James and De Jong (1961).

The present work describes improvements to the Zimmermann reaction which have been developed over the last few years in these laboratories, the objectives being increased sensitivity, reproducibility and, especially, the production by straightforward means of a resultant colour which is free from inter-

${ }^{1}$ Postal address: Institute of Clinical Pathology and Medical Research, Post Office Box 108, Lidcombe, New South Wales, Australia.

Received for publication 27 April 1962. ference by extraneous chromogens, thus eliminating the need for the usual background corrections or for colour extraction.

\section{METHOD}

The following reagents are required:-

PURIFIED METHANOL A good grade of methanol is refluxed for three to four hours with $2 \mathrm{~g}$. per litre of 2:4-dinitrophenylhydrazine and a few drops of concentrated sulphuric acid, and then distilled with an efficient splash-head. A Quickfit anti-foam head is effective. With less efficient heads a second distillation may be necessary to remove small amounts of the hydrazine. An atmosphere of nitrogen is used throughout, although this is not essential. The product, once free from aldehydes and ketones, keeps well under ordinary conditions. Purification is essential.

ETHYLENE DICHLORIDE The British Drug Houses product is left to stand overnight with 8-16 g. of phosphorus pentoxide per litre. The following day it is distilled once. Some workers use the commercial solvent directly without further treatment.

$m$-DINITROBENZENE $1 \%$ The product supplied by British Drug Houses for ketosteroid determinations is suitable without further purification. It is dissolved in the purified methanol. The solution keeps well when stored in an amber glass bottle away from light and under refrigeration.

POTASSIUM HYDROXIDE $6.4 \mathrm{M}$ Fresh potassium hydroxide pellets (A.R. grade), 225 g., are dissolved as rapidly as 
possible and with good shaking in about $350 \mathrm{ml}$. of purified methanol, the solution cooled, diluted to $500 \mathrm{ml}$. with the same solvent, and mixed. The reagent is allowed to stand overnight and then decanted from deposited carbonate or filtered through sintered glass of fairly fine porosity. The molarity is then adjusted to 6.4 to $6.5 \mathrm{M}$ by titration of a carefully measured aliquot of the somewhat viscous solution (use a 'to contain' pipette wiped on the outside with tissue after filling; run the alkali into distilled water and rinse the pipette by sucking the diluted alkali up and down in the pipette several times). The reagent keeps well for long periods when stored in completely filled polythene bottles and kept in the refrigerator. The reagent in daily use declines slowly in normality because of uptake of carbon dioxide (see below).

STEROID STANDARD Dehydroisoandrosterone, $3.0 \mathrm{mg}$., is dissolved in purified methanol, the solution diluted to $100 \mathrm{ml}$. with that solvent, mixed, and transferred to a polythene bottle. This reagent keeps for long periods.

The following procedure for assay of urinary steroids is carried out:-

STEROID EXTRACTION Orthodox acid hydrolysis is employed for liberating the free steroids. Urine, $10 \mathrm{ml}$, and $3 \mathrm{ml}$. of $10 \mathrm{M}$. hydrochloric acid are mixed and heated in water at $100^{\circ} \mathrm{C}$. for eight minutes. The hydrolysate is cooled, $10 \mathrm{ml}$. of ethylene dichloride is added, and the mixture is shaken mechanically for 15 minutes. After centrifugation the top aqueous layer is removed by suction and the organic layer filtered through Whatman No. 1 paper into a tube which can be glass-stoppered. Fifteen to 20 sodium hydroxide pellets are added, the tube stoppered and phenolic and acidic substances removed by shaking for 15 minutes. This is followed by filtration through Whatman No. 42 paper. Aliquots, each of $2 \mathrm{ml}$., of the filtrate in test tubes are then evaporated to dryness in water at $100^{\circ} \mathrm{C}$. The procedure described is, of course, for the assay of 17-ketosteroids. In order to determine 17-ketogenic and 17-hydroxycorticosteroids, preliminary bismuthate oxidation without or with prior borohydride reduction is carried out by the methods of Norymberski et al. and Appleby et al., using $5 \mathrm{ml}$. of urine for the latter determinations. The remainder of the procedure is essentially as for 17 -ketosteroid assay.

COLOUR DEVELOPMENT Equal volumes of a $m$-dinitrobenzene $1 \%$ and $6.4 \mathrm{M}$ methanolic potash are mixed in sufficient quantity for immediate needs. Of this mixed reagent $0.8 \mathrm{ml}$. is added to the dried extract prepared as described, the tube thoroughly agitated to dissolve the residue, and the solution incubated for 10 minutes in a closed water bath at $37^{\circ} \mathrm{C}$. It is then removed, cooled to room temperature, and diluted with $5 \mathrm{ml}$. of ethanol, the water content of which is not critical. This ethanol does not need purification and any good quality commercial product may be employed.

Standards are prepared by evaporating to dryness suitable volumes of the standard steroid solution and then developing as for the extract. One standard at least is always included, in duplicate, with each set of assays.

Two blanks are prepared, each in duplicate. The first is a reagent blank, developed in plain tubes; the second is a method blank, produced by processing 10 or $5 \mathrm{ml}$. of water in lieu of urine. Normally neither of these should give readings exceeding $\sim 3 \%$ of full-scale deflection for the meter of a colorimeter, when measured against water. The method blank is usually very little higher than the reagent blank, the latter giving about $8 \%$ of the colour produced by a $30 \mu \mathrm{g}$. standard, when both are measured against a water reference.

Readings are made at $530 \mathrm{~m} \mu$ with a spectrophotometer, or by means of a colorimeter with an Ilford $\mathbf{6 2 5}$ filter. The resultant from the urine extract is measured against the method blank and that from the standard against the reagent blank. The colour is stable for about 25 minutes at room temperature, after which the absorbance decreases linearly at the rate of about $10 \%$ per hour. Since standards and tests decrease proportionately there is no critical period during which the colorimetry should be performed, but from the sensitivity point of view the time should not be too protracted. No turbidity problems have been encountered during or after colour development.

\section{EXPERIMENTS AND RESULTS}

In early investigations highly purified $m$-dinitrobenzene up to $2 \%$ concentration, ethanol purified as for methanol, and methanolic potassium hydroxide up to $7 \mathrm{M}$ were used. Variation of the parameters verified that the major portion of the interfering brown background absorption stems from the reagents employed and not from the urinary extract. Although at higher reagent concentrations the sensitivity was increased, the blanks were so large as to preclude the use of these higher levels of $m$-dinitrobenzene and alkali. The complete elimination of ethanol at the reaction stage, and its replacement by methanol, gave immediate improvement. The brown background no longer appeared, even at high reagent concentrations, being replaced by a weaker yellow colour which had very little absorbance in the region of maximum absorbance of the steroid reaction product.

Variations in the diluting solvent after development showed only minor changes in blanks and sensitivity; no advantage was gained from using purified methanol as diluent. Ethanol in concentrations from 70 to $100 \%$, with water as the second component, is satisfactory.

VARIATIONS IN REAGENT CONCENTRATIONS Decreases in the strength of the methanolic alkali below about 6.4 M led to appreciable decreases in sensitivity and slightly lower blanks at a $m$-dinitrobenzene concentration of $0.5 \%$ (in methanol); at the $1 \%$ level the 
effect was the same, but less marked. With the alkali concentration constant at $6.4 \mathrm{M}$ little difference in sensitivity was observed when the $m$-dinitrobenzene concentration was varied over the range 0.5 to $1 \%$. However, as the molarity of the potassium hydroxide reagent in daily use tends to drop with time, the $1 \%$ level of $m$-dinitrobenzene was retained. In practice, the conditions are thus somewhat flexible. Any decrease in molarity of the alkali with time may be compensated for by increasing its proportion in the mixed reagent described under 'Method'. The original ratio of $1: 1$ may be increased stepwise to $3: 2$. Although this results in a somewhat lower concentration of $m$-dinitrobenzene in the mixture, this is more than offset by the increase in final concentration of alkali. In this way reagents may be conserved.

DEVELOPMENT TEMPERATURE At $60^{\circ} \mathrm{C}$. the colour developed was found to be rapidly destroyed. This was not the case at $37^{\circ} \mathrm{C}$. In four minutes some $90 \%$ of maximum colour was produced from a $30 \mu \mathrm{g}$. standard and in 10 minutes the maximum had been reached. After 16 minutes there was a small increase in the blank when measured against water, but no significant change in the standard when read against the blank.

OTHER STEROIDS Only two others were examined for chromogenicity relative to dehydroisoandrosterone. One, aetiocholanolone, gave approximately the same colour equivalent as the standard substance. The other was 17a,21-dihydroxypregna-1,4-diene-3, 11,20-trione, a compound closely related to cortisone. This failed to give any colour under the conditions described, in spite of three keto groups at sites other than the 17-position. As far as it goes, this would suggest a fair degree of specificity for 17-keto function, but other steroids would need to be examined to confirm or refute this observation. Certainly, it has been well established that under varying conditions the Zimmermann reaction leads to different molar absorbancies at a given wavelength for different steroids containing a 17-keto function; but while this is of paramount importance for the estimation of individual steroids, it is less so for the 'collective' types of assay usually carried out. The steroid excretion pattern varies so much from person to person in a manner which is usually unknown, that in the present state of knowledge there seems little point in attempting a thorough assessment of the chromogenic power of numbers of individual steroids.

CALIBRATION The absorbance of the resultant was found to be proportional to the concentration of dehydroisoandrosterone up to a level equivalent to about $30 \mu \mathrm{g}$. of standard. Above this level there $\stackrel{S}{\rightarrow}$ was a relative decrease in absorbance, a $90 \mu \mathrm{g}$. standard reading about $6 \%$ lower than the value calculated from that for $30 \mu \mathrm{g}$. Attempts to obtain linearity by adding alkali or acid to the diluent after $\stackrel{\otimes}{\triangle}$ reaction were unsuccessful. With $m$-dinitrobenzene at the $2 \%$ level and a reaction time of 16 minutes at $37^{\circ} \mathrm{C}$. a virtually linear plot is obtained but at the expense of a higher blank, and we prefer therefore to use the conditions described under 'Method'. For raised levels of urinary steroids it is thus necessary for accurate work to include a higher standard or to repeat the assay with a smaller volume of extract.

EXAMINATION FOR BACKGROUND ABSORBANCE Extraneous chromogens may be defined as any contributing absorbance in thespectrum from the extract having an absorption spectrum different from that of the pure standard. In general, if no background of this kind exists, then $r_{\lambda}$, the ratio of the absorbance of the test spectrum $\left(A_{t}\right)_{\lambda}$ to that of the standard $\left(A_{s}\right)_{\lambda}$ at any wavelength $\lambda$, will be constant when $\lambda$ is varied. The interval of wavelength over which this variation is considered will be that surrounding the region of maximum absorbance, since greatest sensitivity will occur here. If any extraneous colour is present, as defined above, it can be shown readily that $r_{\lambda}$ will pass through a maximum, minimum, or a number of these, as $\lambda$ varies continuously. If, however, the range of variation $r_{\lambda}$ is small, and is, in addition, sensibly constant over a given region, preferably near the absorption maximum of the standard spectrum, then it may be concluded that within this region there is very little, if any, interference. The results obtained for the present method (with $\lambda_{\max }$ at $520 \mathrm{~m} \mu$ ) are illustrated by the example in Table I.

It is seen that for a typical analysis, $r_{\lambda}$ varies by only $6 \%$ over the range $480-600 \mathrm{~m} \mu$ and is almost constant throughout the interval $520-600 \mathrm{~m} \mu$, indicating negligible interference from non-steroidal material. It was found that the interval of least variation in $r_{\lambda}$ was $530-560 \mathrm{~m} \mu$, that is at wavelengths slightly higher than $\lambda_{\max }$. For this reason $530 \mathrm{~m} \mu$ is perhaps to be preferred as the wavelength for measurement in the method described.

As regards the reproducibility of determinations Table II gives data for duplicate determinations on $30 \mu \mathrm{g}$. standards and on urinary extracts. The data were taken sequentially and represent the results of routine assays carried out over a period of weeks with changes of operator and reagents. The results are expressed as colorimeter readings.

Standard errors of measurement (S.E.M.) were 
TABLE I

VARIATION IN $\mathbf{r}_{\lambda}$ WITH SPECTRAL WAVELENGTH

\begin{tabular}{llll}
$\lambda$ & $A_{T}$ & $A_{S}$ & $r_{\lambda}$ \\
\hline 440 & 0.113 & 0.113 & 1.00 \\
460 & 0.148 & 0.148 & 1.00 \\
480 & 0.195 & 0.210 & 0.93 \\
500 & 0.244 & 0.268 & 0.91 \\
520 & 0.270 & 0.300 & 0.90 \\
540 & 0.255 & 0.290 & 0.88 \\
560 & 0.230 & 0.263 & 0.875 \\
580 & 0.193 & 0.219 & 0.88 \\
600 & 0.147 & 0.164 & 0.895 \\
620 & 0.103 & 0.110 & 0.935
\end{tabular}

TABLE II

DUPLICATE DETERMINATIONS OF $30 \mu \mathrm{g}$. STANDARDS AND OF URINE EXTRACTS UNDER ROUTINE CONDITIONS

\begin{tabular}{|c|c|c|c|}
\hline \multicolumn{2}{|l|}{ Standards } & \multicolumn{2}{|l|}{ Tests } \\
\hline Colorimeter Readings & $\Delta \%$ & Colorimeter Readings & $\Delta \%$ \\
\hline $\begin{array}{l}25 \\
23\end{array}$ & $4 \cdot 2$ & $\begin{array}{l}16 \cdot 8 \\
18 \cdot 4\end{array}$ & $4 \cdot 5$ \\
\hline $\begin{array}{l}25 \\
24 \cdot 6\end{array}$ & 0.8 & $\begin{array}{l}16 \cdot 8 \\
16 \cdot 2\end{array}$ & 1.8 \\
\hline $\begin{array}{l}23 \\
21 \cdot 8\end{array}$ & $2 \cdot 7$ & $\begin{array}{l}9.4 \\
8.9\end{array}$ & 2.2 \\
\hline $\begin{array}{l}22.5 \\
20.5\end{array}$ & $4 \cdot 7$ & $\begin{array}{l}75 \\
68\end{array}$ & 4.9 \\
\hline $\begin{array}{l}21 \\
23\end{array}$ & $4 \cdot 5$ & $\begin{array}{l}28 \\
25 \cdot 5\end{array}$ & $4 \cdot 7$ \\
\hline $\begin{array}{l}20 \\
21\end{array}$ & $2 \cdot 4$ & $\begin{array}{l}14.2 \\
15.8\end{array}$ & $5 \cdot 3$ \\
\hline $\begin{array}{l}23 \\
22\end{array}$ & $2 \cdot 2$ & $\begin{array}{l}23 \\
22\end{array}$ & $2 \cdot 2$ \\
\hline $\begin{array}{l}22.9 \\
21 \cdot 2\end{array}$ & $4 \cdot 1$ & $\begin{array}{l}29 \cdot 5 \\
30\end{array}$ & 0.8 \\
\hline $\begin{array}{l}24 \cdot 3 \\
24 \cdot 3\end{array}$ & $\mathbf{0}$ & $\begin{array}{l}17 \cdot 0 \\
17 \cdot 2\end{array}$ & 0.6 \\
\hline $\begin{array}{l}23.5 \\
24 \cdot 5\end{array}$ & $2 \cdot 1$ & $\begin{array}{l}25 \\
25 \cdot 5\end{array}$ & $1 \cdot 0$ \\
\hline $\begin{array}{l}22 \cdot 4 \\
22 \cdot 4\end{array}$ & 0 & $\begin{array}{l}13 \cdot 2 \\
12 \cdot 8\end{array}$ & $1 \cdot 5$ \\
\hline $\begin{array}{l}22.4 \\
22.0\end{array}$ & 0.9 & $\begin{array}{l}15.7 \\
12.7\end{array}$ & $10 \cdot 6$ \\
\hline $\begin{array}{l}23 \\
24\end{array}$ & $2 \cdot 1$ & $\begin{array}{l}7.5 \\
8.5\end{array}$ & $6 \cdot 3$ \\
\hline
\end{tabular}

calculated from these data by means of the relationship

$$
(\text { S.E.M. })^{2}=\frac{\sum \triangle^{2}}{2 \mathrm{~N}}
$$

where $\Delta=$ percentage difference between the mean value of a pair of duplicates and the value of either of them,

and $\mathbf{N}=$ number of pairs of duplicate determinations.

This gave for the standards a S.E.M. of $\pm 2.0 \%$, and for the tests, one of $\pm 3.1 \%$.

\section{DISCUSSION}

One of the major embarrassments in the estimation of 17-ketosteroids by the Zimmermann reaction has been the development of a considerable amount of extraneous brown colour from both standards and extracts. In some modifications at least, the amount appearing in the extracts is greater than in the standards so that correction for the extraneous colour is essential. The fact that it appears in the resultants from pure standards indicates a weakness in the reaction systems commonly employed. Of the attempts which have been made to compensate for extraneous chromogens, that described by Allen (1950) is frequently used. This consists in subtracting from the absorbance at or near the maximum an amount equal to the average absorbancies at two wavelengths equally spaced on each side of the original wavelength. That is, a linear background is assumed. Such corrections are not entirely satisfactory for several reasons. In the first place background absorption is rarely linear (except for electronic spectra with sharp absorption bands); and secondly, inaccuracies result with the broad-banded type of spectrum usually encountered in colorimetry because of insufficient differences between the absorbancies at the three wavelengths selected. Further, the wider the wavelength intervals chosen in order to overcome this inaccuracy, the less valid becomes the assumption of linear background; thus one error is replaced by another.

Purification of the Zimmermann product has also been employed (see James and De Jong, 1961, for references and discussion) by taking advantage of the differing partitions of colour and background interference between two immiscible phases, frequently aqueous-ethanol and ether. Ideally, the procedure should achieve complete isolation of the Zimmermann chromogens in the ethereal phase and of the extraneous colour in the aqueous-ethanol phase. In practice, neither of these objectives is quite attained.

There are also the further disadvantages of an additional step in the assay and of quantitative errors arising from the use of such volatile extractants as ether. For the Zimmermann reaction carried out under any given set of conditions, the final colour could theoretically be composed of three contributions, namely the Zimmermann chromogens, any increased blank colour in the urine extract over and above that in the method blank, and lastly, any other non-steroidal chromogens from the urine extract. In order to assess the magnitude of these contributions, and at the same time the effectiveness of ether extraction, a blank, extract, and standard were reacted with our reagents as usual. Instead of then diluting with ethanol, $2 \mathrm{ml}$. of $30 \%$ ethanol was added followed by $6 \mathrm{ml}$. ether. The tubes were then stoppered and shaken vigorously for about half 
a minute. The spectra of the ethereal layers were measured. The lower aqueous phases were diluted with ethanol and their spectra measured also. Analysis of the curves showed that the Zimmermann chromogens were not quite completely extracted and that some of the blank colour was extracted by ether. The blank contribution in the urine extract was only slightly higher than the blank, and no contribution from other non-steroidal substance could be found. It appears therefore that the method described measures Zimmermann chromogens almost exclusively, any variation in blank contributions at $530 \mathrm{~m} \mu$ being so small as to be negligible.

For future work it would be of interest to combine the advantage of both organic bases and methanolic reagents; in this way, blanks should be virtually zero, reagents completely stable, and calibration linear.
We wish to thank Professor W. Klyne and his staff for the supply of steroids from the Steroid Reference Collection of the Medical Research Council. Approval for $\overrightarrow{0}$ publication of this work has been given by the Director of State Health Services, New South Wales Department of Public Health.

\section{REFERENCES}

Allen, W. M. (1950). J. clin. Endocr., 10, 71.

Appleby, J. I., Gibson, G., Norymberski, J. K., and Stubbs, R. D. (1955). Biochem. J., 60, 453.

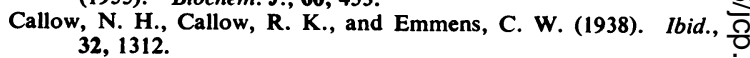

Hickinbottom, W. J. (1936). Reactions of Organic Compounds, $\vec{G}$ p. 322. Longmans, Green and Co., London.

James, V. H. T., and De Jong, Mary (1961). J. clin. Path., 14, 425. Norymberski, J. K. (1952). Nature (Lond.), 170, 1074.

, Stubbs, R. D., and West, H. F. (1953). Lancet, 1, 1276.

Zimmermann, W. (1935), Hoppe-Seylers Z physiol Chem., 257.

-, (1936). Ibid., 245, 47.
Editorial

The conflict of living matter with the mineral world: The pneumoconioses A. POLICARD

Studies on the character and staining of fibrin A. C. LENDRUM, D. S. FRASER, W. SLIDDERS, and R. HENDERSON

The kidney in rheumatic heart disease DOROTHY S. RUSSELL

Observations on the incidence, aetiology, and pathology of senile osteoporosis R. A. CALDWELL

Cystadenoma and cystadenocarcinoma of the pancreas J. A. CAMPBELL and A. H. CRUICKSHANK

Fatal recurrence of parathyroid carcinoma after seven years J. P. CALEY, E. E. JONES, and D. $\mathrm{H}$. COLLINS

Platelet aggregation J. R. O'BRIEN Part I Some effects of the adenosine phosphates, thrombin, and cocaine upon platelet adhesiveness Part II Some results from a new method of study

Serum vitamin $B_{12}$ in renal failure D. M. MATTHEWS and A. GORDON BECKETT
The estimation of red cell volume with ${ }^{51} \mathrm{Cr}$-labelled erythrocytes and plasma volume with radioiodinated human serum albumin D. HART and J. METZ

Diagnostic tests for thyroid antibodies: A comparison of the precipitin and latex-fixation (Hyland TA) tests J. R. ANDERSON, W. W. BUCHANAN, R. B. GOUDIE, and K. G. GRAY

A simple latex slide test for rheumatoid arthritis using the gamma globulin in the patient's serum R. BIRD and R. G. BURR

Serum protein fractions in rheumatoid pneumoconiosis without arthritis

R. B. PAYNE

Biochemical classification of Klebsiella correlated with the severity of the associated disease W. D. FOSTER and JEAN BRAGG

An epidemic of gastroenteritis due to an uncommon variant of Eschericia coli 0.128 W. I. H. SHEDDEN, C. W. POTTER, V. DUBOWITZ, and MARGARET WOOD

Technical method

A method for the electrophoresis of colicines in agar gel PaUl J. CHAPPLE

Book reviews

Copies are still available and may be obtained from the PUBLISHING MANAGER, BRITISH MEDICAL ASSOCIATION, TAVISTOCK SQUARE, W.C.I, price 17s. 6D. 